\title{
Measurement of the diffusion coefficient in liquids using Fresnel diffraction from a phase step
}

\author{
Ahad Saber ( $\square$ ahad.saber@gmail.com ) \\ University of Mohaghegh Ardabili Faculty of Basic Sciences https://orcid.org/0000-0002-8879-9878 \\ Mohammad Taghi Tavassoly \\ University of Tehran
}

\section{Rasoul Aalipour}

Azarbaijan Shahid Madani University Faculty of Basic Sciences

\section{Research Article}

Keywords: Fresnel diffraction, diffusion coefficient, diffusion

Posted Date: May 3rd, 2021

DOl: https://doi.org/10.21203/rs.3.rs-453639/v1

License: (c) (1) This work is licensed under a Creative Commons Attribution 4.0 International License.

Read Full License

Version of Record: A version of this preprint was published at Applied Physics B on July 5th, 2021. See the published version at https://doi.org/10.1007/s00340-021-07655-0. 
myjournal manuscript No.

(will be inserted by the editor)

\title{
Measurement of the diffusion coefficient in
}

\section{liquids using Fresnel diffraction from a phase}

\section{step}

\author{
Ahad Saber ${ }^{1}$, Mohammad Taghi Tavassoly ${ }^{2}$, Rasoul Aalipour ${ }^{3}$ \\ 1 Department of Physics, University of Mohaghegh Ardabili, PO Box 179, Ard- \\ abil, Iran \\ 2 Physics Department, University of Tehran, KargarShomally, Tehran 14395-547, \\ Iran \\ 3 Department of Physics, Azarbaijan Shahid Madani University, Tabriz 53714- \\ 161, Iran
}

Received: date / Revised version: date

\begin{abstract}
Fresnel diffraction from a phase step for measuring diffusion coefficient in transparent liquids is investigated. When a transparent glass plate immersed vertically in a cell containing two diffusing liquids is illuminated by a parallel beam of light, the diffraction pattern of the plate edge forms on a screen perpendicular to the beam direction and varies by diffusion. The gradient of the refractive index in the liquids is then obtained by
\end{abstract}

Send offprint requests to: Ahad Saber 
analyzing the diffraction patterns at different times after the beginning of the diffusion process, from which the diffusion coefficient is determined. Using this method, we study the diffusion process of the sucrose-water solution and report the diffusion coefficient with a reliable accuracy.

\section{Introduction}

Diffusion proces is ubiquitous in nature, science, and technology, ranging from pollution control, chemical and mechanical engineering, to crystal growth, biological systems and separation of isotopes [1-3]. Among various chemical and physical methods that are used to study this process in transparent media, the most important ones are the optical methods, which mainly are based on measuring the refractive index or its derivative variation $[4,5]$. Interferometry methods such as Michelson interferometry [6], Mach-Zehnder interferometry [7], electronic speckle pattern interferometry (ESPI) [8], digital holographic interferometry [9], and the moiré technique [10] have been used to study the diffusion process. Besides the fact that the interferometry techniques are noninvasive, and provide real-time results, their main advantage is they that are highly accurate and reliable. There are however some drawbacks for the interferometry techniques, the most important of which are their sensitivity to mechanical noises, their experimental setup complications, and the difficulties in the measuring procedures. 
A widely used method in derivation of the diffusion coefficient is determining the distance of the peaks in concentration difference profile between two times. Also, some other methods including tracing the interference fringes and fitting the concentration profile equations to the experimental data at different times have been developed [9].

Recently it has been shown that discontinuous changes in phase or phase gradient of the light beam leads to an appreciable diffraction that is referred to as Fresnel diffraction from phase steps $[11,12]$. This effect was studied theoretically and experimentally $[13,14]$, and applied to a plenty interesting metrological measurements. The measurements include refractive indices of materials with high accuracy [15-18], nonlinear refractive index [19], thickness of thin films and plates [20], nanometer displacement [21], wavelength, and spectral line profile $[17,22]$.

In this paper, we introduce a new method for the study of the diffusion process based on the Fresnel diffraction from a phase step in transmission. The refractive index profile of the diffusing liquids is determined using the Fresnel diffraction pattern of the edge of a glass plate immersed vertically inside a diffusion cell. Then the diffusion coefficient of liquids is obtained using the refractive index profile at two different time. This method has all the advantages of the interferometry techniques, besides, practically is not sensitive to vibrations and can be applied easily. 

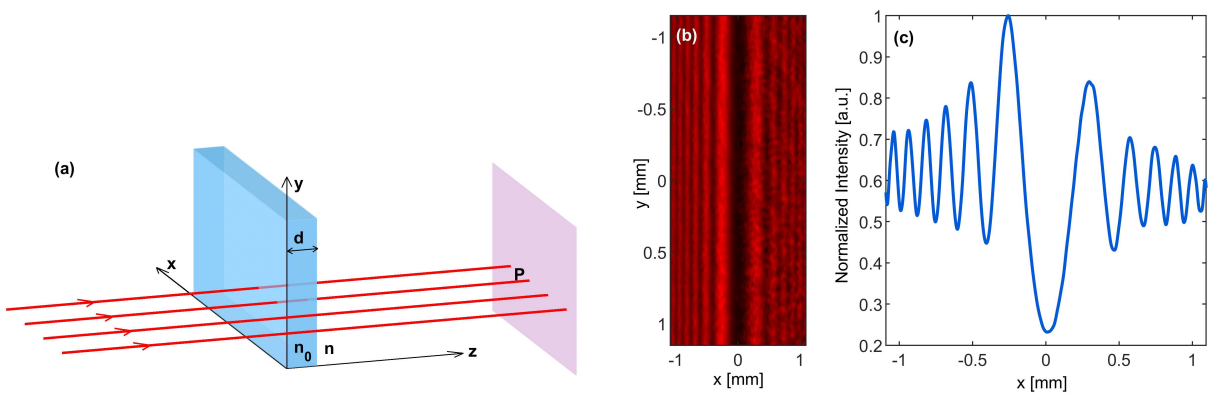

Fig. 1 (a) A transparent plate with refractive index $n_{0}$ and thickness $d$ is illuminated by a parallel beam of light in the neighborhood of its edge in a medium with refractive index $n$, (b) the resulted Fresnel diffraction pattern formed on a screen perpendicular to the beam direction, and (c) the corresponding intensity profile.

\section{Theory}

In Fig. 1(a), a parallel beam of light strikes the edge area of a glass plate of the thickness $d$ and refractive index $n_{0}$. The transmitted light forms a diffraction pattern on a screen perpendicular to the beam direction. The normalized intensity on the diffraction pattern at arbitrary point $\mathrm{P}$ is given by $[11,13]$

$$
I_{n}(P)=\cos ^{2}\left(\frac{\phi}{2}\right)+2\left(C_{0}^{2}+S_{0}^{2}\right) \sin ^{2}\left(\frac{\phi}{2}\right)+\left(C_{0}-S_{0}\right) \sin \phi
$$

where $\phi$ is the phase change at the plate edge, and $C_{0}$ and $S_{0}$ are the Fresnel cosine and sine integrals [23]. A typical diffraction pattern of the plate edge and the corresponding intensity profile are shown in Figs. 1(b) and $1(\mathrm{c})$, respectively. Here, we consider a free diffusion process in one dimension that causes refractive index gradient in a specified direction. The 
free diffusion process is characterized by a constant diffusion coefficient, $D$, and described by Fick's second law

$$
\frac{d C(y, t)}{d t}=D \frac{d^{2} C(y, t)}{d y^{2}}
$$

where $C(y, t)$ is the concentration at position $y$ and time $t$. The solution of Eq. (2) for the mixture of two liquids with concentrations $C_{1}$ and $C_{2}$, initially separated at $y=0$ is given by [24]

$$
C(y, t)=\frac{C_{1}+C_{2}}{2}+\frac{C_{1}-C_{2}}{2} \operatorname{erf}\left(\frac{y}{2 \sqrt{D t}}\right)
$$

where $\operatorname{erf}(x)$ is the error function. For small concentration ranges, the relation between the concentration and the time-dependent refractive index can be considered linear as follows [5]

$$
n(y, t)=\left(\frac{d n}{d c}\right)_{0} C(y, t)+n^{\prime}
$$

where $\left(\frac{d n}{d c}\right)_{0}$ stands for the mean slope of the refractive index curve versus concentration in the applied concentration range and $n^{\prime}$ is a constant. Therefore, we can consider the refractive index distribution inside the diffusion cell by

$$
n(y, t)=\frac{n_{1}+n_{2}}{2}+\frac{n_{1}-n_{2}}{2} \operatorname{erf}\left(\frac{y}{2 \sqrt{D t}}\right),
$$

where $n_{1}$ and $n_{2}$ are the refractive indices corresponding to concentrations $C_{1}$ and $C_{2}$, respectively. Now, denoting the refractive index difference at two different times as

$$
\Delta n\left(y, t_{2}, t_{1}\right)=n\left(y, t_{2}\right)-n\left(y, t_{1}\right)
$$


and, using Eq. (5) and Eq. (6) we get

$$
\Delta n\left(y, t_{2}, t_{1}\right)=\frac{n_{1}-n_{2}}{2}\left(\operatorname{erf}\left(\frac{y}{2 \sqrt{D t_{1}}}\right)-\operatorname{erf}\left(\frac{y}{2 \sqrt{D t_{2}}}\right)\right)
$$

The time-dependent phase of the light beams passing through the edge area of the plate inside the diffusion cell is related to the refractive index as follows

$$
\phi(y, t)=\frac{2 \pi}{\lambda}\left(n(y, t)-n_{0}\right) d
$$

where $\lambda$ is the wavelength of the incident light. The variation of the refractive index during the diffusion process changes the phase difference along the plate edge. Therefore, using Eq. (8), we can write

$$
\Delta \phi(y, t)=\frac{2 \pi d}{\lambda} \Delta n(y, t)
$$

By derivating from Eq. (9),

$$
\frac{d\left(\Delta \phi\left(y, t_{2}, t_{1}\right)\right)}{d y}=0
$$

and doing some calculations, two extremums are obtained as follows

$$
y_{1,2}= \pm \sqrt{\frac{2 D \ln \left(t_{2} / t_{1}\right)}{\left(1 / t_{1}\right)-\left(1 / t_{2}\right)}} .
$$

Eq. (11) realizes the relation between the extremums of the phase difference and the diffusion coefficient. Therefore, by measuring the distance between two consecutive extremums, $\Delta y=y_{2}-y_{1}$, the diffusion coefficient can be derived from the following equation:

$$
D=\frac{\Delta y^{2}\left[1 / t_{1}-1 / t_{2}\right]}{8 \ln t_{2} / t_{1}} .
$$




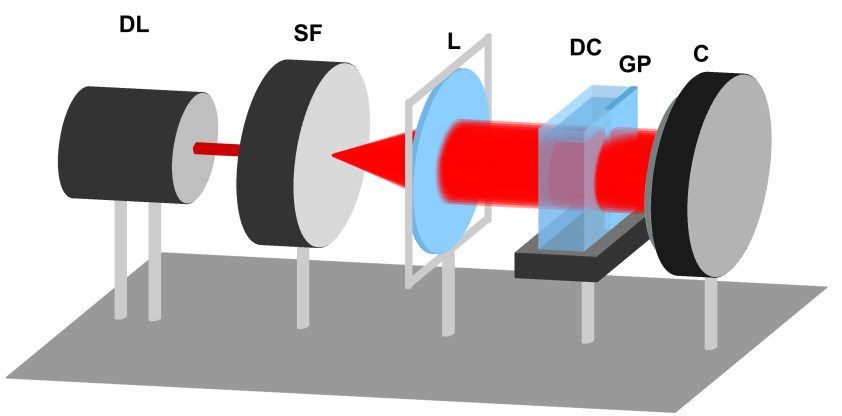

Fig. 2 The scheme of the experimental setup, in which DL, SF, L, DC, GP, and C represent diode laser, spatial filter, collimating lens, diffusion cell, glass plate, and camera, respectively.

\section{Experimental Results}

The sketch of the experimental setup is presented in Fig. 2. A diode laser beam of wavelength $\lambda=655 \mathrm{~nm}$ is expanded and collimated using a spatial filter SF and a lens L. The beam is transmitted through a transparent rectangular cell of dimensions $25 \times 36 \times 36 \mathrm{~mm}^{3}$, DC, containing the diffusion solution. The heavier solution ( $3 \%$ sucrose--water solution) is introduced from below of the lighter solution (distilled water) using a capillary tube to avoid any convection current. (distilled water on the top of $3 \%$ sucrosewater solution). A glass plate of thickness $2 \mathrm{~mm}$, GP, is installed inside the cell, perpendicular to the beam direction. The diffraction pattern of the light diffracted from the edge of the plate is recorded by a camera (Nikon D5200, $6000 \times 4000$ resolution, and $3.9 \mu \mathrm{m}$ pixel pitch) every 5 minutes. 


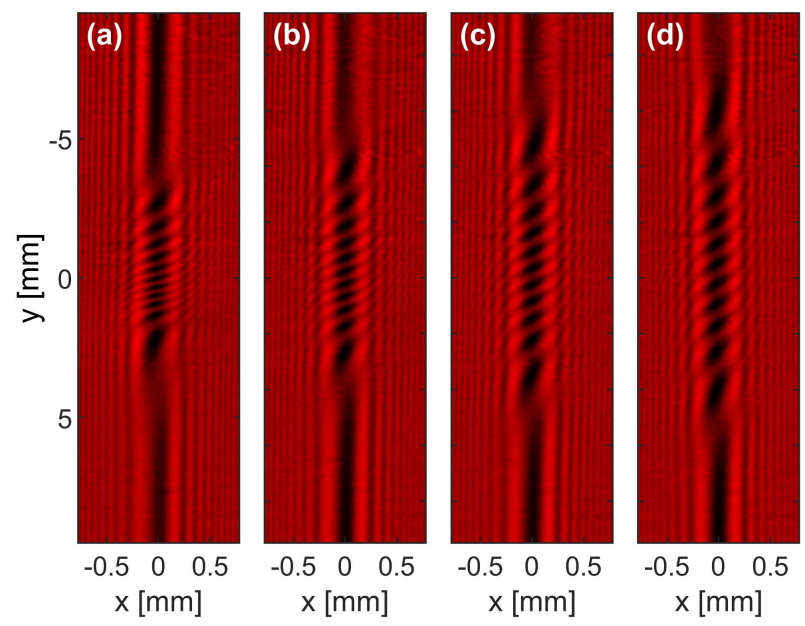

Fig. 3 The diffraction patterns of the light diffracted from the edge of a glass plate immersed inside the diffusion cell containing distilled water on the top of $3 \%$ sucrose-water solution at times (a) 30, (b) 60, (c) 100, and (d) 150 minutes after the beginning of the diffusion process.

Figs. 3(a)-(d) show the diffraction patterns at times, $t=30,60,100$ and 150 min after the beginning of diffusion process. The corresponding intensity profiles of the diffraction patterns along the edge of the plate are plotted in Figs. 4(a)-(d). According to the plots, the intensity distribution along the edge is almost periodic and the fringe spacing increases by the time of diffusion, though the number of fringes remains constant until the diffusion process approaches to the ends of the cell. In this situation, the initial assumptions for existing the solution of the form expressed in Eq. (3) are confirmed. 


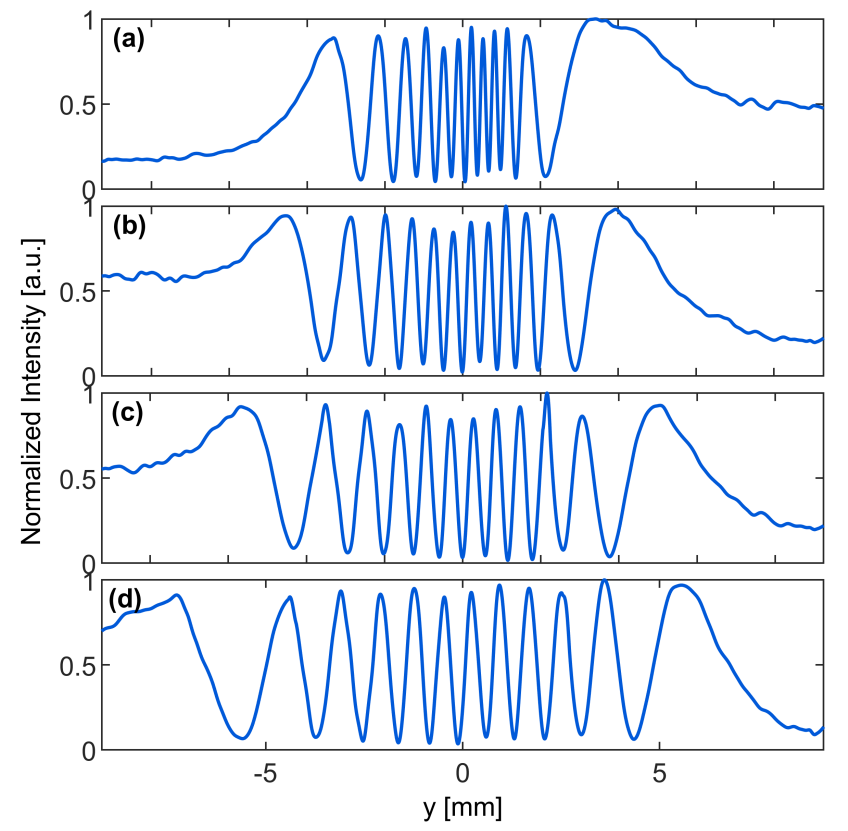

Fig. 4 The intensity profiles of the diffraction fringes along the edge of the plate, at times (a) 30, (b) 60, (c), 100, and (d)150 minutes after the beginning of the diffusion process.

For points on the edge line, $\mathrm{x}=0$, Eq. (1) reduced to

$$
I_{n}(y, t)=\frac{1}{2}(1+\cos \phi(y, t))
$$

where the phase change is a function of $y$ and $t$. According to the theoretical considerations, for determining the diffusion coefficient, we need to evaluate the phase, $\phi(y, t)$. For this purpose, we applied the Fourier transform method which is implemented using the Fast Fourier transform (FFT) algorithm $[25,26]$. Fig. 5 shows the unwrapped phase profiles that correspond to the intensity profiles in Figs. 4(a) and 4(c), which have been obtained at times 30 and 100 minutes after beginning of the diffusion process. By 


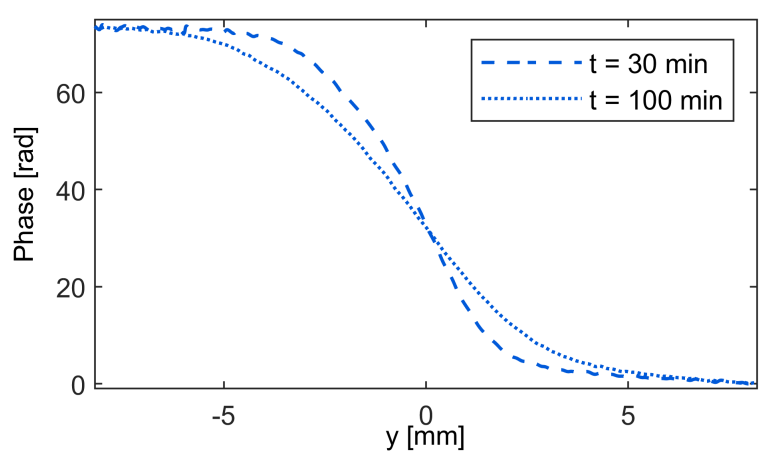

Fig. 5 The unwrapped phase profiles of the diffraction fringes along the plate edge at times, $\mathrm{t}=30$ and $\mathrm{t}=100$ minutes, after the beginning of the diffusion process. subtracting the phase profiles at these times we get the phase difference, illustrated in Fig. 6. The diffusion coefficient is deduced from the distance between the consecutive extremums of the phase difference profile, according to the Eq. (12). By the way, the diffusion coefficient for several time intervals is calculated and presented in Table 1 . The mean value has been derived $(0.54 \pm 0.03) \times 10^{-5} \mathrm{~cm}^{2} \mathrm{~s}^{-1}$, which is comparable with the standard value of $0.52 \times 10^{-5} \mathrm{~cm}^{2} \mathrm{~s}^{-1}$ for low concentrations at $25^{\circ} \mathrm{C}$ [27]. It should be noted that the uncertainty in the measurement of the diffusion coefficient is derived by calculating the standard deviation of the measured values illustrated in Table 1.

\section{4 conclusion}

The Fresnel diffraction from the phase step is applied to determine the diffusion coefficient in transparent solutions. It is considerably simpler than other optical methods such as interferometry, which requires very accurate 


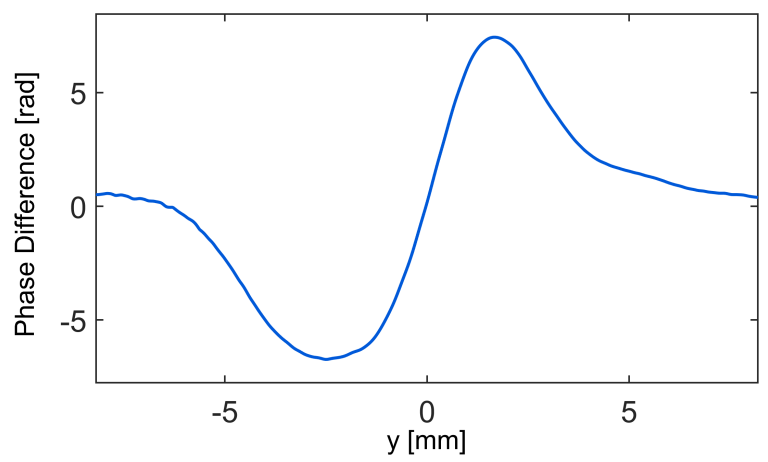

Fig. 6 The plot of the phase difference associated with the phase profiles in Fig. 5.

Table 1 The diffusion coefficients deduced for different time intervals after beginning the diffusion process and their mean value.

\begin{tabular}{c|c|c|c}
$t_{1}(\mathrm{~min})$ & $t_{2}(\mathrm{~min})$ & $D \times 10^{5}\left(\mathrm{~cm}^{2} \mathrm{~s}^{-1}\right)$ & $D_{\text {mean }} \times 10^{5}\left(\mathrm{~cm}^{2} \mathrm{~s}^{-1}\right)$ \\
\hline 30 & 60 & 0.54 & \\
40 & 70 & 0.50 & \\
50 & 80 & 0.52 & \\
30 & 70 & 0.54 & $0.54 \pm 0.03$ \\
60 & 100 & 0.57 & \\
40 & 90 & 0.52 & \\
40 & 100 & 0.54 & \\
40 & 110 & 0.55 &
\end{tabular}

optics. Furthermore, this method is almost not sensitive to mechanical vibrations, which is the main drawback of the interferometry methods. It is suitable for a wide range of concentrations. Like other optical methods, it 
is limited to transparent liquids. In a case that one deals with the solutions with the small difference of concentrations, it is suggested to use a wedge instead of the plate and analyze the diffraction fringes.

\section{References}

1. Edward Lansing Cussler. Diffusion: mass transfer in fluid systems. Cambridge university press, 2009.

2. Juan F Torres, Atsuki Komiya, Eita Shoji, Junnosuke Okajima, and Shigenao Maruyama. Development of phase-shifting interferometry for measurement of isothermal diffusion coefficients in binary solutions. Optics and Lasers in Engineering, 50(9):1287-1296, 2012.

3. Henry John Valentine Tyrrell and KR Harris. Diffusion in liquids: a theoretical and experimental study. Butterworth-Heinemann, 2013.

4. Gyanendra Sheoran, Arun Anand, and Chandra Shakher. Lensless fourier transform digital holographic interferometer for diffusivity measurement of miscible transparent liquids. Review of scientific instruments, 80(5):053106, 2009.

5. D Ambrosini, D Paoletti, and Nasser Rashidnia. Overview of diffusion measurements by optical techniques. Optics and Lasers in Engineering, 46(12):852-864, 2008.

6. Vani K Chhaniwal, Arun Anand, and CS Narayanamurthy. Measurement of diffusion coefficient of transparent liquid solutions using michelson interferometer. Optics and Lasers in Engineering, 42(1):9-20, 2004. 
7. Elvio E Alanı, Graciela G Romero, Carlos C Martinez, et al. Interferometric measurement of diffusion coefficients through a scanning laser beam. Optical Engineering, 39(3):744-750, 2000.

8. David Karlsson, Guido Zacchi, and Anders Axelsson. Electronic speckle pattern interferometry: a tool for determining diffusion and partition coefficients for proteins in gels. Biotechnology progress, 18(6):1423-1430, 2002.

9. MG He, S Zhang, Y Zhang, and SG Peng. Development of measuring diffusion coefficients by digital holographic interferometry in transparent liquid mixtures. Optics express, 23(9):10884-10899, 2015.

10. Kazem Jamshidi-Ghaleh, Mohammad Taghi Tavassoly, and Nastaran Mansour. Diffusion coefficient measurements of transparent liquid solutions using moiré deflectometry. Journal of Physics D: Applied Physics, 37(14):1993, 2004 .

11. M Amiri and MT Tavassoly. Fresnel diffraction from $1 \mathrm{~d}$ and $2 \mathrm{~d}$ phase steps in reflection and transmission modes. Optics communications, 272(2):349-361, 2007.

12. Ehsan A Akhlaghi, Ahad Saber, and Zahra Abbasi. Fresnel diffraction due to phase gradient singularity. Optics letters, 43(12):2840-2843, 2018.

13. M Taghi Tavassoly, Mohammad Amiri, Ahmad Darudi, Rasoul Aalipour, Ahad Saber, and Ali-Reza Moradi. Optical diffractometry. JOSA A, 26(3):540-547, 2009.

14. Hamid Salvdari, M Taghi Tavassoly, and SR Hosseini. Fresnel diffraction from a step in the general case. JOSA A, 34(4):674-680, 2017.

15. M Taghi Tavassoly and Ahad Saber. Optical refractometry based on fresnel diffraction from a phase wedge. Optics letters, 35(21):3679-3681, 2010. 
16. M Taghi Tavassoly, Roxana Rezvani Naraghi, Arashmid Nahal, and Khosrow Hassani. High precision refractometry based on fresnel diffraction from phase plates. Optics letters, 37(9):1493-1495, 2012.

17. M Taghi Tavassoly, Seyed Roohollah Hosseini, Ali Motazedi Fard, and Roxana Rezvani Naraghi. Applications of fresnel diffraction from the edge of a transparent plate in transmission. Applied optics, 51(30):7170-7175, 2012.

18. Arash Sabatyan and Mohammad Taghi Tavassoly. Application of fresnel diffraction to nondestructive measurement of the refractive index of optical fibers. Optical Engineering, 46(12):128001-128001, 2007.

19. Masood G Beygi, Rouhollah Karimzadeh, and Masoomeh Dashtdar. Nonlinear refractive index measurement by fresnel diffraction from phase object. Optics \& Laser Technology, 66:151-155, 2015.

20. Mohammad Taghi Tavassoly, Iman Moaddel Haghighi, and Khosrow Hassani. Application of fresnel diffraction from a phase step to the measurement of film thickness. Applied optics, 48(29):5497-5501, 2009.

21. Ali Akbar Khorshad, Khosrow Hassani, and Mohammad Taghi Tavassoly. Nanometer displacement measurement using fresnel diffraction. Applied optics, 51(21):5066-5072, 2012.

22. SR Hosseini and MT Tavassoly. The application of a phase step diffractometer in wavemetry. Journal of Optics, 17(3):035605, 2015.

23. Max Born and Emil Wolf. Principles of optics: electromagnetic theory of propagation, interference and diffraction of light. Elsevier, 2013.

24. John Crank et al. The mathematics of diffusion. Oxford university press, 1979.

25. Daniel Malacara. Optical shop testing, volume 59. John Wiley \& Sons, 2007. 
26. Charles Van Loan. Computational frameworks for the fast Fourier transform, volume 10. Siam, 1992.

27. Norberto Bochner and Jacob Pipman. A simple method of determining diffusion constants by holographic interferometry. Journal of Physics D: Applied Physics, 9(13):1825, 1976. 


\section{Figures}
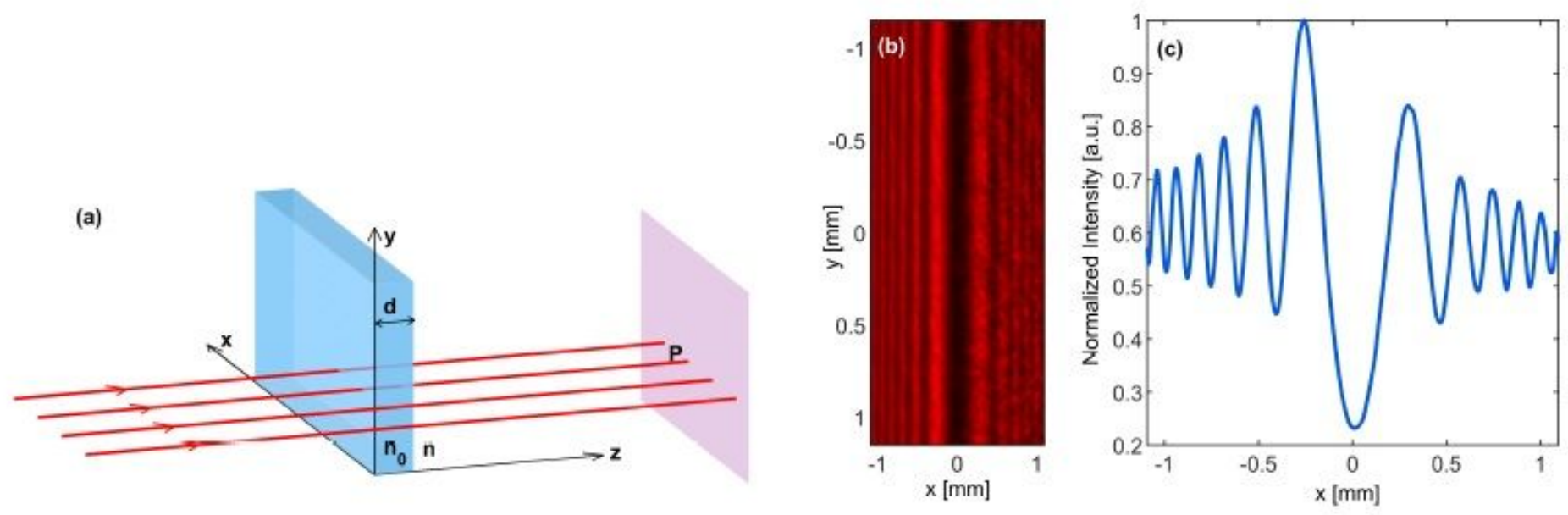

Figure 1

(a) A transparent plate with refractive index $\mathrm{n} 0$ and thickness $\mathrm{d}$ is illuminated by a parallel beam of light in the neighborhood of its edge in a medium with refractive index $n$, (b) the resulted Fresnel diffraction pattern formed on a screen perpendicular to the beam direction, and (c) the corresponding intensity prole.

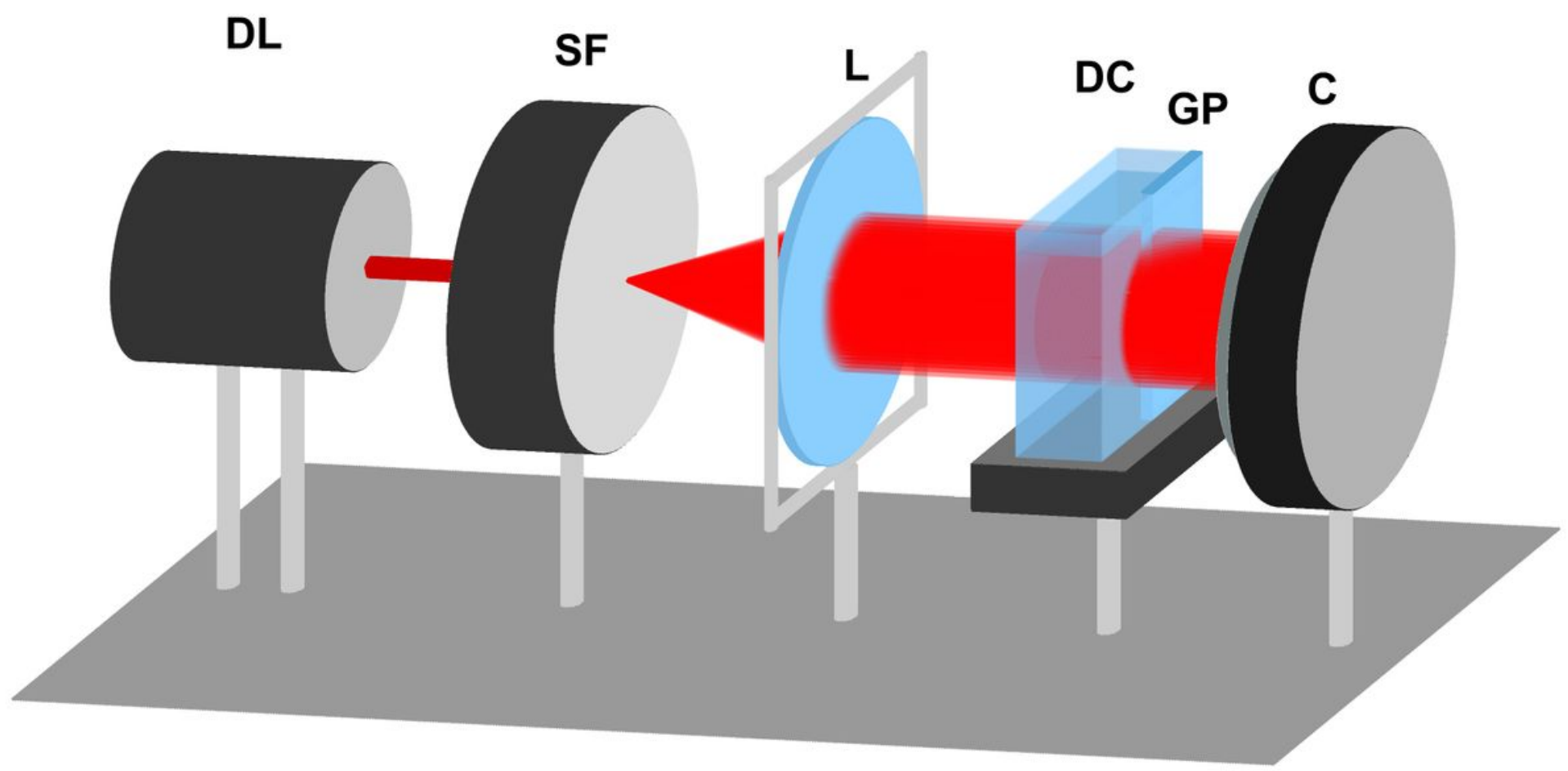

Figure 2 
The scheme of the experimental setup, in which DL, SF, L, DC, GP, and C represent diode laser, spatial filter, collimating lens, diffusion cell, glass plate, and camera, respectively.
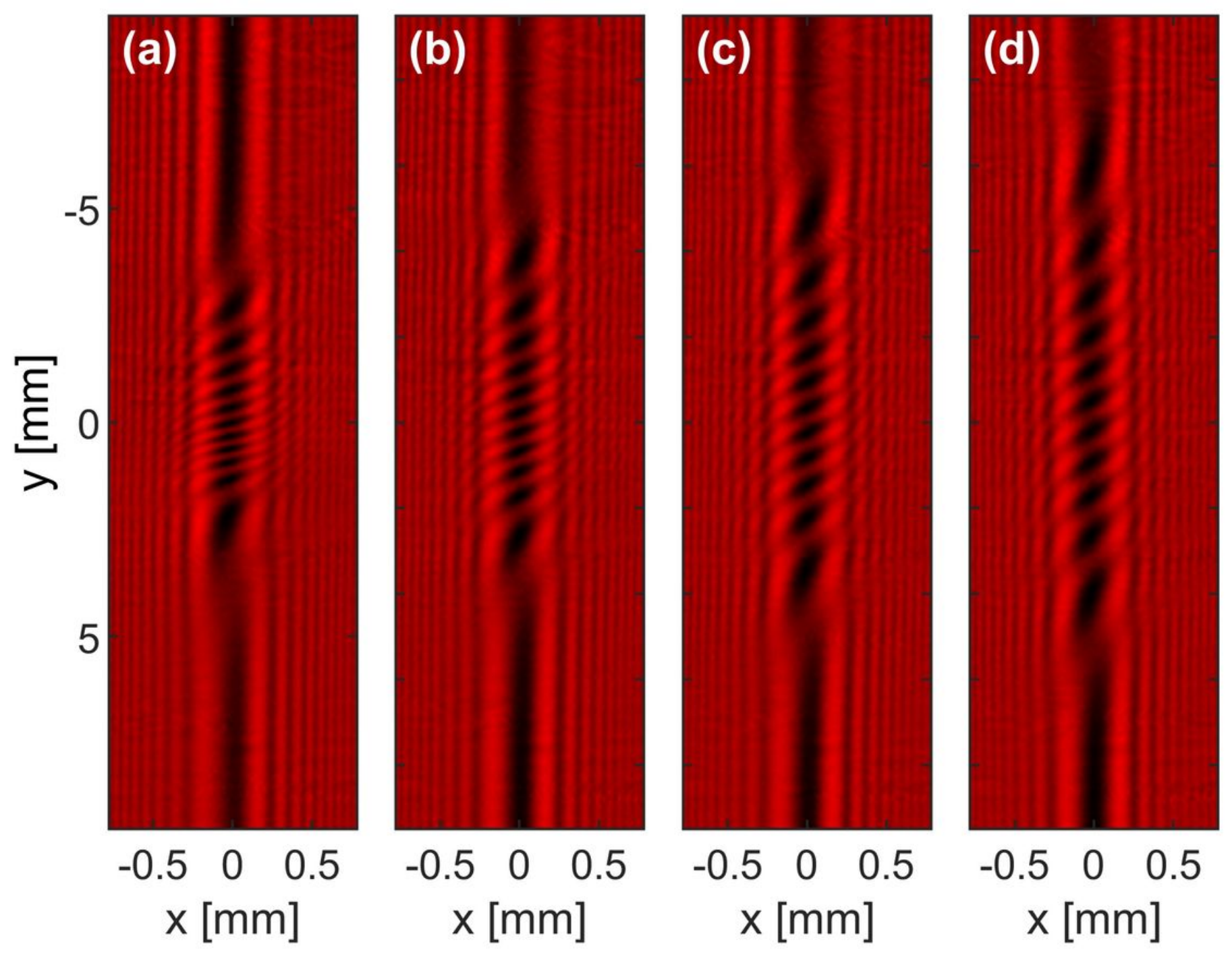

Figure 3

The diffraction patterns of the light diffracted from the edge of a glass plate immersed inside the diffusion cell containing distilled water on the top of $3 \%$ sucrose-water solution at times (a) 30 , (b) 60 , (c) 100 , and (d) 150 minutes after the beginning of the diffusion process. 


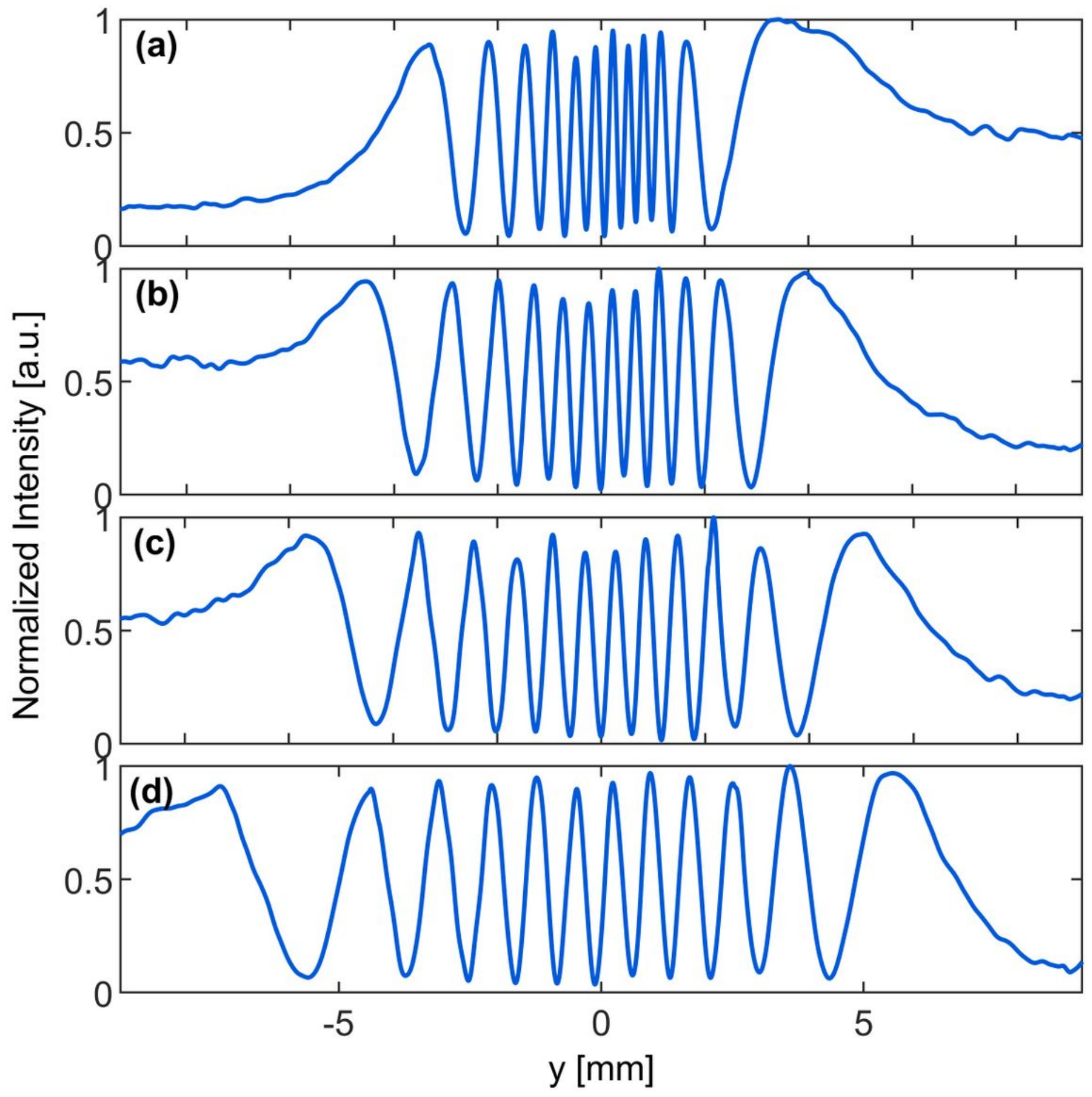

Figure 4

The intensity proles of the diffraction fringes along the edge of the plate, at times (a) $30,(b) 60,(c), 100$, and (d) 150 minutes after the beginning of the diffusion process. 


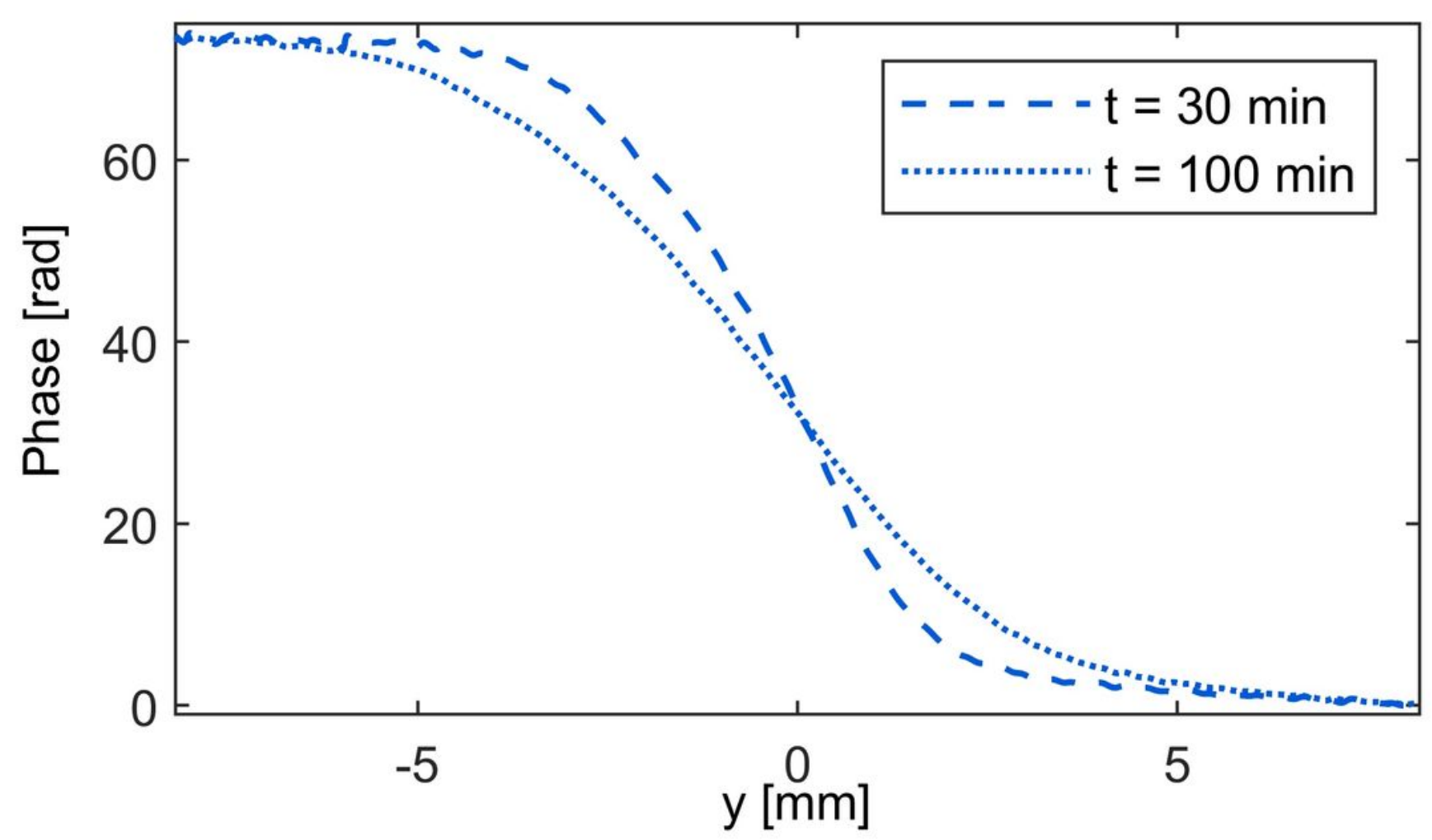

Figure 5

The unwrapped phase proles of the diffraction fringes along the plate edge at times, $t=30$ and $t=100$ minutes, after the beginning of the diffusion process.

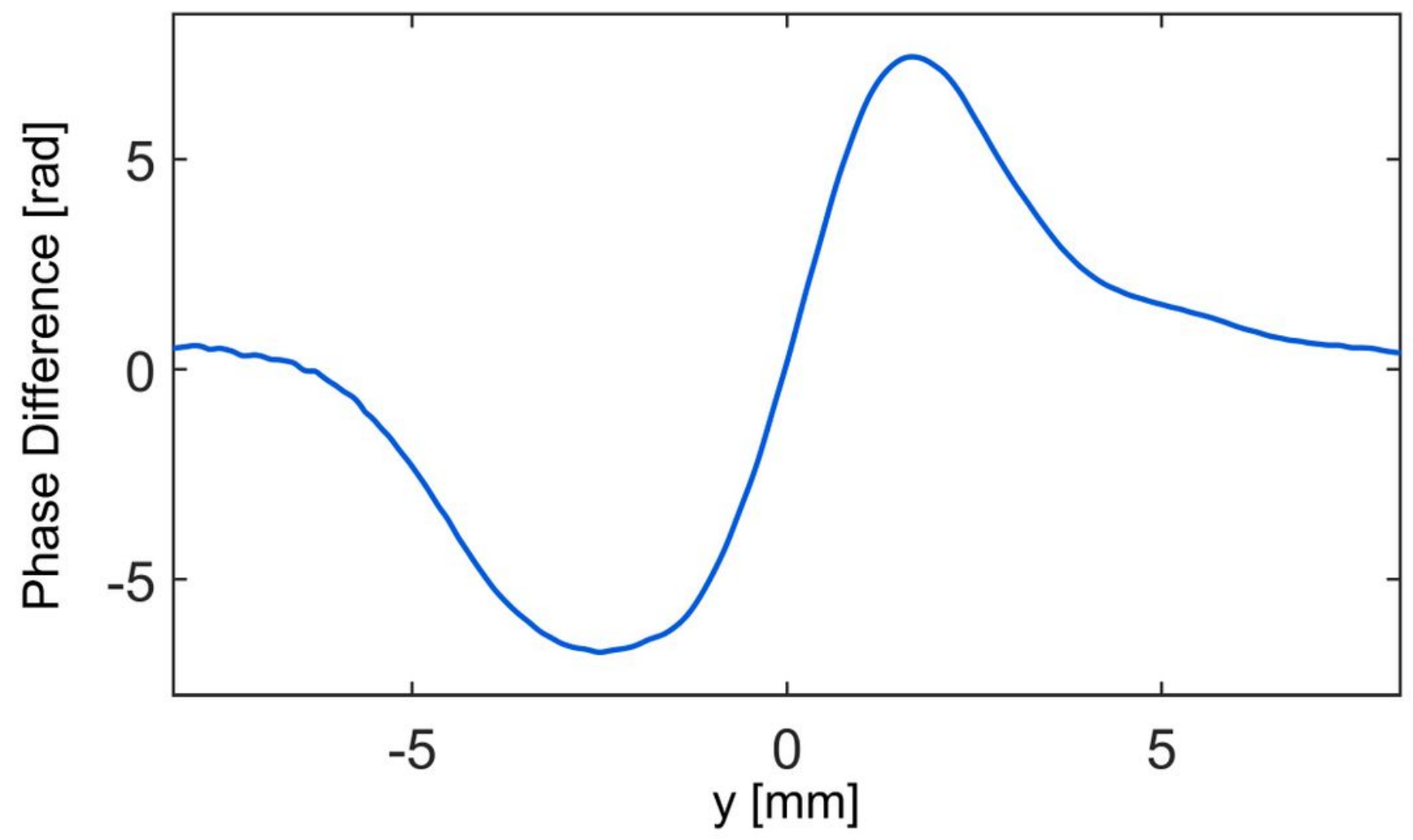


Figure 6

The plot of the phase difference associated with the phase proles in Fig. 5. 